\title{
Cross-sectional and longitudinal studies in the elderly
}

\author{
By A. N. Ехтол-Sмiтн, Department of Geriatric Medicine, St. Pancras Hospital, \\ St. Pancras Way, London NWI oPE
}

In cross-sectional or point-of-time studies, measurements are made on individuals of different ages. Comparison of the results between various age-groups (for example young and old) will reveal age differences. In longitudinal studies serial measurements are made on the same individual at two or more points of time. These studies are the only means of revealing true age changes. Ideally the longitudinal study requires that measurements should be made at standardized intervals throughout the whole life-span. This can be achieved in experimental animals with short life-spans but in humans the duration of the study is likely to be limited to a period of ro-20 years. For this reason this type of study is carried out during phases of life when rapid changes occur, for example after the age of retirement. The Department of Health and Social Security (DHSS) has organized a nutritional survey of the elderly population in six centres in Great Britain, starting in 1967-68 and with subsequent examinations 5 and ro years later. The results of analyses on a cross-sectional basis have already been published (DHSS, 1972, 1979) and the report on the 10-year longitudinal study is in preparation.

\section{Methodological problems}

In all epidemiological studies in the elderly there are the initial problems of locating the elderly and the choice of the most appropriate sample. Furthermore, information collected must be based on social history, measurements of dietary intake, medical examination, anthropometry and laboratory investigations.

Locating the elderly. Gilmore \& Caird (1972) have discussed four possible sources of information on which a register of the elderly can be compiled. The two favoured methods are:

1. The use of records of Local Health Executive County Councils which contain the names of people over the age of $6_{5}$ years on the lists of general practitioners within the National Health Service. Since a medical examination is essential in epidemiological nutritional studies of the elderly population it is necessary to have the collaboration of general practitioners. This method, therefore, has much to commend it and almost the entire population can be covered, but there is known to be inaccuracy in the Local Health Executive Council lists.

2. The Register of Electors prepared for the census which is conducted every ro years gives the names and addresses of people over the age of 18 years but it does not distinguish those over the age of 65 years.

Gilmore \& Caird (1972) have shown that a combination of these two sources is satisfactory. The inaccuracy of the Health Executive Council lists can be reduced from $3 \mathrm{r} \%$ to $6 \%$ by the use of the Electoral Roll as a check. The price of this 
increase in accuracy and the associated saving in time resulting from the avoidance of wasted visits is the omission of a small proportion of the elderly $(2-3 \%$ at the most) from the survey. In most instances this small loss is acceptable.

Nature of the sample. It is desirable in large-scale nutritional surveys of the elderly population that the sample should cover the whole country and should be representative of both urban and rural living. This is necessary to take account of the differences due to racial, occupational, socio-economic and cultural factors as well as variations dictated by customs in different parts of the country.

Random sample. Ideally a truly random sample should be used but in many instances it will have to be very large. Thus if a characteristic in the population has a normal distribution of values then $95 \%$ of the data will be collected from individuals within $\pm 2 \mathrm{SD}$ of the mean, but it may well be that the individuals most worthy of study are the $5 \%$ within the tails of the distribution.

Cluster analysis. To overcome this problem an alternative sampling technique can be used when studying the influence of environmental factors. It has been shown (P. R. Payne, unpublished results) from an analysis of data derived from the 1971 census that it is possible to define certain characteristics of the elderly population in rural and urban areas in England and Wales. Of twenty variables investigated the following were found to be correlated: the death rate in the population, the elderly death rate, the percentage in socio-economic groups 6 and over, and the percentage of houses with basic facilities. A factor can be calculated which takes account of the relative weighting of these contributions. The 172 towns, boroughs and country districts in England and Wales can be placed in ranking order according to the value of this factor. A high negative value represents a 'good' environmental area with low mortality patterns and a high positive value a 'bad' environmental area. In the DHSS Nutrition Survey of the elderly, which was carried out in $1973-74$, the areas chosen for the survey included those at the extremes of the distribution of characteristics comprising this formula.

Weighting the sample. If the sample is randomly drawn from all ages over $6_{5}$ years the number of very old people, for example over the age of 90 years, will be small and often insufficient to give the required level of significance in statistical tests when comparisons are made with younger ages. This problem can be overcome by weighting the sample with respect to age. For example, by including equal numbers of subjects within the two age-groups $65-74$ years and 75 years and above. In view of the differential in longevity between males and females it may also be desirable to introduce similar weightings in respect of the sex distribution.

Response. In all epidemiological studies in the elderly, active collaboration of the subjects is required and this may not be forthcoming in those elderly people who are socially isolated, apathetic, depressed or mentally-impaired; in fact from those individuals who may be deemed most worthy of study. A high proportion of non-responders will therefore bias the sample and is likely to exclude those subjects who are most at risk of developing malnutrition.

Social history. Many social, domestic and economic changes occur in the lives of elderly people after retirement and these may have a profound influence on 
nutritional status. Thus adequate social assessment by experienced observers is essential. The social and occupational classification which can be applied to populations of working age may not be so readily applicable to the elderly, particularly wives and widows. Thus only crude assessments of socio-economic groupings can be made. Environmental aspects are especially important in longitudinal studies since any age-associated changes revealed by the study may be incorrectly attributed to the effects of ageing when in fact they are due to alterations in the domestic and economic circumstances which must almost inevitably occur with advancing age and during the course of long-term studies. Thus true age changes may be confounded with the effects due to environmental changes.

Dietary investigations. There are several techniques by which dietary intakes can be measured but some of these are not suitable for nutritional surveys of the elderly. In practice a compromise has often to be reached between accuracy and the extent to which elderly subjects can comply with the techniques. The method used in the DHSS Nutrition Surveys (1972, 1979) is to establish the normal pattern of intake by means of a dietary history and in a subsequent 7 -d-period to measure the food intake by weighing. Nutrient intakes are calculated from food tables. Since there is marked seasonal variation in the intake of some nutrients (for example, vitamin C) it is important that the survey should cover the whole period of I year. Moreover, in longitudinal studies, in order to avoid the confounded effects of seasonal variation, subjects must be investigated in successive surveys at corresponding times of the year.

Medical examination. Several studies of the health of the elderly population at home have revealed a high prevalence of disease and disability. In one such study (Exton-Smith, 1977) it was found that only about one-third of the subjects were fit and the remaining two-thirds suffered from significant diseases and disability which could profoundly influence nutritional status. The high incidence of multiple pathology has been demonstrated in the epidemiological studies of the elderly population in Edinburgh conducted by Williamson et al. (1964) who found that men of 65 years and over have a mean of 3.26 diseases and the women a mean of 3.42 diseases. Moreover, there is a striking increase in the incidence of disease with age and of the use of drugs for the treatment of these diseases. These findings are relevant in cross-sectional studies when the observations on very old people are compared with those in a younger age-group (for example, 65-69 years) and in longitudinal studies where impairment of function and disability increase progressiveiy during the course of the study.

Further difficulties arise in large-scale cross-national surveys since the use of several clinical investigators is unavoidable. It is rarely possible to avoid inter-observer errors as different clinicians adopt different criteria in their assessment of health. Furthermore, there are difficulties in making a clinical diagnosis of malnutrition; definite signs and symptoms of clear-cut deficiencies are readily recognized but the diagnosis becomes more difficult when malnutrition is marginal and where non-nutritional disease, which is so common in older people, 
produces manifestations indistinguishable clinically from those due to nutritional deficiency. In some cases where malnutrition is suspected it may not be possible to confirm the diagnosis unless the subject is willing to submit to more detailed investigations which are not usually within the scope of epidemiological studies.

Laboratory investigations. In order to eliminate possible errors due to differences in techniques used in different laboratories it is usual in national surveys to undertake biochemical and haematological investigations in one laboratory. This can present problems in the transport of specimens and it excludes certain investigations such as estimation of leucocyte ascorbic acid which must be carried out within hours of collection of the blood sample. In such instances when different laboratories must be used the need for quality control and standardization is essential. Ideally the time of collection of blood specimens from subjects participating in the different centres must also be standardized in order to minimize the effects due to diurnal variations and to the ingestion of food.

\section{Analysis and interpretation of results}

Cross-sectional studies. In general, cross-sectional epidemiological studies of the nutrition of the elderly population have been useful in achieving the following objectives: (a) The assessment of the nutritional status of the elderly and comparing it with that of younger adults. (b) The identification of malnutrition and of risk factors associated with its causation. (c) The establishment of normal values or reference ranges for biochemical, haematological, anthropometric and other information, with particular relevance to those related to nutrition.

Cross-sectional surveys, however, do not accurately describe the course of development or of ageing of individuals since the observed age differences may be due to the operation of several factors. These include the effects of ageing (i.e. true age changes), the effects of disease (pathological ageing), group effects and social and environmental influences (pseudo-ageing). Analysis of the results of cross-sectional studies rarely indicates the relative contributions of each of these variables. The most serious disadvantage, however, of this type of study is the intrusion of group effects.

I. Some of the differences are due to differences in date of birth of subjects surveyed, since in developing societies young and old people are from very different cultural backgrounds and it is known that dietary patterns are profoundly influenced by socio-economic and cultural factors.

2. Disease and disability have been shown to have a greater influence on nutrient intake than the effects of ageing alone (DHSS, 1972, 1979). Further evidence that low intakes of nutrients are associated with impairment of health which leads to reduced physical activity has been obtained from a study of housebound old people in which their intakes were compared with age-matched active old people (Exton-Smith et al. 1972). By contrast, Debry et al. (1977) have shown in studies in farm workers in the Lorraine region in France that those who maintain their active mode of life to an advanced age also maintain their nutrient intakes; decline in intake with age which is seen 
in urban dwellers does not occur in the majority of active farmworkers in rural areas.

3. Some of the age effects in cross-sectional studies may be obscured by the phenomenon known as 'survival of the fittest'. Thus in extreme old age the studies may reveal an apparent increase in some survey variable even when the overall trend with age is downwards. Individuals who survive to the age of 85 years and beyond represent the 'biological elite' with characteristics which enable them to outlive their former contemporaries. The phenomenon has been observed in relation to ageing bone and it has been found that men over the age of 85 years have an apparently greater bone mass than those aged 75 years (Exton-Smith, 1970).

Longitudinal studies. These studies represent the best means of detecting the effects of true age changes in nutritional status. The results of these studies fail to reveal any reversal in any overall downward trend with age which may be seen in cross-sectional studies. Techniques for the analysis of results of longitudinal studies have only recently been evolved. A method which is commonly employed is as follows. The absolute values representing change in a variable are converted into a percentage change; this percentage change is then converted to an annual percentage change using the compound growth formula, and the annual percentage change is then related to the age of each person at his or her age midway between the two points in time over which the observations are made. Calculations show that for some variables the rate of change with age is constant whereas for others a curvilinear relationship is found with a slowing of the rate of change with advancing age. Another analytical method for calculating the results of longitudinal studies is by the use of cusum plots. This technique has been used by A. Horsman and colleagues (unpublished results) in examining the relationship between vitamin $D$ status and the rate of bone loss in an elderly population. The summated change in metacarpal cortical widths, which is used as a measure of the quantity of bone, is plotted against the summated period of observation (subject years) when the subjects are arranged in rank order according to the value of another measured variable (in this case the plasma concentration of 25 -hydroxycholecalciferol). The dependence of bone loss on vitamin $\mathrm{D}$ level can be ascertained by relating significant changes in the slope of the curve to the 25-hydroxycholecalciferol values.

\section{Conclusions}

For obvious resons few longitudinal studies of the health and nutrition of elderly populations have been conducted and the results of the DHSS longitudinal studies covering a period of to years are awaited with interest. In the future it seems likely that more attention must be paid to the possibilities of combining cross-sectional and longitudinal studies. A comparison of the results derived from the two types of study would seem to offer the best means of separating those changes due to ageing and those due to disease, group effects and environmental and 
socio-economic influences that invariably take place during the course of long-term studies.

\section{REFERENCES}

Debry, G., Bleyer, R. \& Mannin, J. M. (1977). Fournal of Human Nutrition 31, 195-203.

Department of Health and Social Security (1972). A Nutritional Survey of the Elderly. Report on Public Health and Medical Subjects no. 3. London: H.M. Stationery Office.

Department of Health and Social Security (1979). Nutrition and Health in Old Age. Report on Health and Social Subjects no. I6. London: H.M. Stationery Office.

Exton-Smith, A. N. (1970). Experimental Gerontology 5, 273-280.

Exton-Smith, A. N. (1977). In Care of the Elderly: Meeting of the Challenge of Dependency, pp. 4I-53 [A. N. Exton-Smith and J. Grimley Evans, editors]. London: Academic Press.

Exton-Smith, A. N., Stanton, B. R. \& Windsor, A. C. M. (1972). Nutrition of Housebound Old People. London: King Edward's Hospital Fund.

Gilmore, A. J. \& Caird, F. I. (1972). Age and Ageing 1, 30-32.

Williamson, J., Stokoe, I. H., Gray, S., Fisher, M., Smith, A., McGhee, A. \& Stephenson, E. (1964). Lancet i, I I I 7-I I 20. 